\title{
Motivating Students to Learn Science: A Physicist's Perspective
}

\author{
M. P. Silverman \\ G. A. Jarvis Professor of Physics, Trinity College, Hartford, USA \\ Email: mark.silverman@trincoll.edu
}

Received 13 September 2015; accepted 27 October 2015; published 30 October 2015

Copyright (C) 2015 by author and Scientific Research Publishing Inc.

This work is licensed under the Creative Commons Attribution International License (CC BY).

http://creativecommons.org/licenses/by/4.0/

(c) (i) Open Access

\begin{abstract}
The objective of this article is to make explicit some concrete ways in which an accurate perspective of what science is contributes significantly to improving science teaching. Effective science teaching begins with the recognition that for both practising scientists and students the desire to find answers to personally meaningful questions about natural phenomena is the strongest incentive to study science. Instructional methods that nurture and draw upon the curiosity of students have the best chance to motivate students to learn science. Teaching in this way entails helping students 1) to see the conceptual relevance, utility, and aesthetic dimension of what they are studying; 2) to appreciate the need for, and power of, rational thinking in problem solving; 3 ) to undertake their own exploratory projects to investigate some aspect of the physical world that interests them. For science teachers to do this well, they must, themselves, a) be knowledgeable of the science they teach, b) keep abreast of advances in their areas of interest, and c) develop their own experimental and observational skills so they can teach with confidence based on personal experience.
\end{abstract}

\section{Keywords}

Science Education, Motivation, Inspiration, Self-Direction, Relevance, Aesthetics, Utility, Rational Thinking, Curiosity, Exploration, Participation

\section{How Science Is Perceived Affects How Science Is Taught}

The best teacher is the one who suggests rather than dogmatises, and inspires his listener with the wish to teach himself.

-Bulwer-Lytton (1873)

The objective of this article is to make explicit some concrete ways in which an accurate perspective of what 
science is contributes significantly to improving science teaching. The substance of my remarks is based on my personal experiences in teaching science (primarily physics, chemistry, environmental sciences) at university and college level, as well as participating in the home-schooling of children from their earliest years through the end of high school. In effect, I have taught science at all levels from primary school through graduate school. Although my concern in this paper focuses on science instruction at college and university level where teachers may also be practising scientists, the principal points I wish to make are of general validity to science education at any level from primary school upward.

Starkly put, the major point is this: science is meaningful only to those who are motivated to study it by their own curiosity.

I am a professional physicist, not a philosopher-and in truth must admit to thinking that philosophers (particularly philosophers of science) have over the past century contributed very little of significance to the progress or understanding of science. Nevertheless, an insightful observation can be found in Alan Watt's selfcharacterisation (Watts, 1970):

A philosopher...is a sort of intellectual yokel who gapes and stares at what sensible people take for granted, a person who cannot get rid of the feeling that the barest facts of everyday life are unbelievably odd. As Aristotle put it, the beginning of philosophy is wonder.

One might add that science, too, begins with wonder, and fostering that wonder should be the paramount task of science teachers at all levels of instruction.

In the sense of curiosity, wonder is the germinal seed of science; there is no science without it, no driving force to uncover the underlying causes and unexpected consequences of those "unbelievably odd" facts of either everyday life or events beyond our immediate perception. But wonder in the sense of the magical, miraculous, and incomprehensible is like a narcotic that destroys curiosity. Herein lies an important distinction between a scientist and the philosopher characterised above: wonder may stimulate both, but scientists go beyond "gapes and stares", employing experimental and mathematical resources to understand in a more profound way the significance of their observations.

Children are born with an innate sense of wonder of both kinds (Fraiberg, 1959). As to curiosity, parents of young children know how difficult it is to prevent them from investigating their world, so intense is this innate drive. I contrast that intensity with the apathy of so many students I have encountered at college and university whose level of interest in the workings of the physical world has diminished almost to the point of extinction. What has happened to these students along their path from childhood to adulthood to so dull the natural inclination to explore and understand their environment? The heart of the matter is identified by educator Jerome Bruner (Bruner, 1966):

The will to learn is an intrinsic motive, one that finds both its source and its reward in its own exercise. The will to learn becomes a "problem" only under specialized circumstances... where a curriculum is set, students confined, and a path fixed. The problem exists not so much in learning itself, but in the fact that what the school imposes often fails to enlist the natural energies that sustain spontaneous learning...

In my view the situation is this: if those who teach science are to accomplish anything, they must stimulate that inborn curiosity without which science itself cannot flourish. Moreover, they must do this under the restrictive conditions of a fixed curriculum that are often responsible in the first place for the diminution of curiosity. Faced with an educational framework over which they may have little control, science teachers can nevertheless influence 1) how they perceive science; 2) what they know of science; and 3) how they interact with their students.

There are links between the perception of science and the teaching of it. The point of view that teachers have on the meaning, content, method, and significance of science in general, and on their own specific involvement in scientific pursuits, all contribute to their effectiveness as teachers. A teacher for whom science is largely a technical discipline will provide a different type of instruction than a teacher for whom science is construed as a cultural activity. A teacher for whom the primary goal of science is the acquisition of accurate data will provide a different type of instruction than a teacher for whom the goal of science is the development of comprehensive theories. Attitudinal differences on the part of the instructor, as in the preceding two examples, may produce students who are interested in science for different reasons, or whose working styles, should they become scientists, are different. However, differences like these are not in themselves educationally harmful. 
Far more serious are perspectives that gravely misconstrue the nature of science as a human activity and method of inquiry. For example:

- A teacher who sees science in terms of authority figures - the alleged great and wise who pass down their knowledge to mere mortals - may well teach science in an authoritarian manner, emphasise and require memorisation of material that scientists themselves would generally look up in references, assign problems involving needless repetition, discourage student inquiries, and repel with indignation student challenges of facts or interpretations.

- A teacher who sees science as a repository of facts from which the correct conclusions ineluctably flow may well communicate to students that personal attributes and human interactions do not matter, that scientific progress follows from slavish adherence to prescribed scientific methods and not from creative imagination and resourceful use of serendipity.

In such classrooms a student's failure to arrive at the right answers could be construed as a sign of mental sluggishness and not of a possibly valid (or at least provocative) alternative way of approaching a proposed problem.

Equally pernicious is the polar opposite perspective whereby the content of science is regarded as merely arbitrary and relative, a product of culture open to any and all modifications and interpretations and expressive of personal biases arising from politics, gender, and other divisive categories. Such may be the case in the so-called social sciences, but it does not describe real sciences like physics, chemistry, geology, and others whose content is validated by replicable experiments and observations, and consistency with well-tested theoretical principles. A teacher who sees science as a collection of equally valid opinions where "anything goes" (Feyerabend, 1975a) and to which anyone irrespective of training and knowledge can contribute cannot possibly communicate to students the seriousness of the enterprise, the importance of accuracy and precision in writing, calculating, and measurement, or the fact that to do well in science requires personal discipline and persistence.

In contrast to the foregoing false perspectives, teachers need to realise that

1) science is a multi-faceted mode of inquiry and not a storehouse of facts; and

2) although science involves personalities, real scientific discoveries inform about the physical world and not about cultural biases.

They can then create in the classroom an atmosphere in which students are encouraged to think, to experiment, to challenge; in short, to engage in the type of creative exploration of which science consists.

\section{What Inspires Scientists Will Inspire Students}

Why should it matter if students (and the adults they become) care little for science? Teachers who do not answer this question satisfactorily early in their science courses lose an opportunity to influence their students' attitudes. For many students who enroll in science courses, the motivation is not so much intrinsic interest as the need to fulfill other requirements such as entry to college or admission to medical school. Science teachers-particularly of subjects like physics, which are perceived to be difficult - therefore often face, at least at the outset, a captive audience to whom the study of science is an imposition, an obstacle to overcome. If students, above all those reluctant to study science in the first place, are to see their science education as a gift and a pleasure, they need to know why science is worth studying.

One answer to the question, which may hardly satisfy a student who dislikes science but which nevertheless is a critically important reason from a societal perspective, is this: Science provides methods, principles, and facts by which to understand and survive (individually and communally) in the real world.

A nation whose citizenry is ignorant of modern science, who relies instead on superstition and ancient scriptures that invoke supernatural guidance, cannot possibly make informed collective judgments on matters pertaining to their vital national interests such as anthropogenic climate change, environmental degradation, dwindling supplies of nonrenewable resources, unsustainable population growth, sensible priorities for medical research, and many other issues. In the US, for example, a study by the US National Science Foundation, released at the 2014 Annual Meeting of the American Association for the Advancement of Science (AAAS), reported that about one half of Americans did not know or believe that human beings evolved from earlier species of animals (Neuman, 2014), and nearly one fourth of Americans did not know that the Earth moved round the Sun (O’Neill, 2014). (The more accurate statement that the Earth and Sun together move round a common centre of mass, was far too advanced a concept even to be queried by the poll.) A study of science literacy in the US from 
a few years earlier presented to the AAAS in 2010 reported that just $28 \%$ of the population (if the sample was representative) improved in science literacy over the preceding two decades (Raloff, 2010). As a nation, the US is noted for its scientific and technological achievements, but these were made by a very small fraction of the population who became scientists and engineers. If the majority of the citizens of a democratic nation were to demonstrate widespread ignorance or denial of basic scientific truths, as reflected in the election of their national leaders and legislative representatives, it is hard to imagine how such a nation could long maintain either a high standard of living or an effective government.

To individual students, however, the socio-political ramifications of caring little for science are probably not foremost on their minds - and so the answer to the question "Why should it matter?" must be a more directly personal one.

Ask research scientists what it is about their work that fascinates them, impels them to pursue it, and rewards them for the countless hours spent at it. What comes across from many such inquiries (e.g. see Sreenivasan, (2004); Conover, (2015)), is the sentiment that science is intellectually exciting, a challenge to one's mental skills; that there is beauty to science, whether in an artful experimental solution to a seemingly insurmountable problem or in the remarkable predictive power of a set of equations. Scientists find satisfaction in operating daily with universal laws and principles applicable everywhere irrespective of cultural differences and geographical separations. Any research scientist can attest to the fact that facility with scientific facts, principles, and experimental and theoretical methods inspires personal confidence to handle problems outside the laboratory as well.

Asked why he is a scientist, Nobel Laureate physicist Murray Gell-Mann responded brusquely: "Because I goddamn well want to know what makes things tick. Period" (Berland, 1962). About a century before GellMann was born, the Scottish physicist James Clerk Maxwell, creator of the unified theory of electricity, magnetism, and light, was less crudely saying the same thing by way of the frequent query: "What's the go o' that?" (Mahon, 2004) Physics, the most fundamental science, has evolved enormously from Maxwell's time to the present, but the primal motivation for why some people become physicists (or scientists in other fields) is largely the same.

To what extent are the stimuli that fire the imagination and motivate the work of professional scientists experienced by students in science classes? Apparently not much, how otherwise to account for why many students think of science as dull and tedious? According to Gell-Mann, the US educational system trains people to study, pass exams, turn in homework, get grades, and please the teacher, but not actually to be or do something (Berland, 1962). The problem is not unique to the US. K. R. Sreenivasan of the International Centre for Theoretical Physics in Trieste wrote (Sreenivasan, 2004).

A paradox of our times is that, while our societies have come to depend on technological advances as never before, the interest in basic sciences is diminishing at all levels.... This state of affairs holds true...in developed as well as developing nations, and deserves our collective attention.

What passes for science education at university level in the US often takes two forms. On the one hand there is instruction that focusses on the quantitative cataloguing, description, and analysis of phenomena with little consideration of significance or aesthetic attraction. On the other hand there is science instruction that forgoes analytical rigour, resorting instead to heuristic explanations that leave the origin and cogency of major results in doubt. In physics the two educational extremes are frequently encountered in introductory courses for physics concentrators and non-science majors, respectively. In the former it is not uncommon to have to pace through a text of more than a thousand pages in two semesters at the rate of a chapter every one or two class sessions. I have rarely found an instructor who enjoys this or who would defend the educational merits of such an approach. But it would seem that nothing can be done. So long as the course syllabus calls for coverage of all of physics, such a pace is thought to be necessary. It is difficult to see how, under such circumstances, a student could achieve anything but the most superficial understanding or experience the intrinsic beauty of the subject.

The physics instruction for nonscientists is not necessarily marked by a lesser flood of facts; indeed the flow may be just as great. But here, in deference to the lesser mathematical abilities or interests of its students, the courses frequently dispense with elements of rigour. Demonstrations that rightfully call for differential or integral calculus are gone; mathematical connections that unify seemingly disparate ideas are also gone. In their stead are qualitative assurances that assertions made by the teacher and textbook are valid. One can understand why students may come to believe that no real explanation exists or that they are intellectually incapable of following it. Often an attempt is made to give relevance to the course by appeal to faddish topics at the fringes of 
plausibility (time travel, "wormholes” through space, multiple universes, and others), even though these topics are totally unrepresentative of what most physicists investigate and are not even subject to feasible experimental verification.

Both educational approaches, in my opinion, are bound to fail to motivate students to study science: the first because a massive edifice of fact does not engage and sustain students' curiosity; the second because students are not inspired to exercise their intellect on subject matter perceived at the outset as defying understanding. The outcome in either case is generally a transient acquisition of unconnected facts and superficial familiarity with catchwords and jargon. Nor is this characteristic of physics alone.

Science teaching is successful, I believe, when students, as active participants and not passive recipients, have the desire to learn, understand the significance of what they are learning, and are motivated to further intellectual growth after their formal education ends. It is successful when it creates in students the same fascination with natural phenomena that is experienced by practising scientists.

How can one provide such an education?

\section{Motivations to Learn Science}

My comments in the preceding section should not be construed to mean that facts in science are unimportant. On the contrary, they are of great importance to scientists. As a physicist who has researched and published in diverse areas of atomic, nuclear, optical, statistical, and gravitational physics, I understand firsthand the need for broad mastery of phenomenological details and mathematical and experimental techniques in science. But—and this point is essential-I acquired such knowledge for specific purposes, namely to solve problems that interested me. As I matured as a professional physicist, the problems that interested me evolved in complexity, subtlety, and diversity, thereby placing greater demands on my need of physical facts and experimental methods. What remained with me at any stage of my development was largely what was personally meaningful, the knowledge I needed to pursue my own particular interests. I believe this to be true not only for learning science, but for the acquisition of knowledge in general.

Certainly, few who study science are expected to become practising scientists. Nevertheless, the cardinal principle of successful science education is the same for all: One expends the effort to learn what is needed to answer questions that are personally meaningful. To realise this is to understand that the essential task of a science teacher is not so much to teach the virtually unlimited details of the subject, but to create in each student an individual need or desire to know. In the course of trying different approaches to teaching physics (Silverman, 1995) at all levels of instruction over a period of nearly five decades, I have come to recognise three principal components to this task:

- Convey to students an accurate and sympathetic impression of what science entails; in particular provide convincing evidence that knowledge of science is an integral part of a civilised, rational culture;

- Help students to develop an ability to think critically, to arrive at logical conclusions after careful acquisition and evaluation of information, to be open-minded, yet able to discern truth from sophistry;

- $\quad$ Provide students an opportunity to develop and implement theoretical knowledge and experimental skills by direct participation in meaningful scientific research.

I consider each component in turn.

\subsection{The Cultural Dimension of Science}

Whereas music, art, and literature can transport people away from the banality or stress of their daily lives to a more pleasurable (or at least less threatening) realm — science, in the minds of many people, is continually probing, discovering, reporting, if not also helping to cause, the preponderance of humanity's anxieties: environmental pollution, climate change, bioterrorism, nuclear terrorism, genetic modifications of the food supply, and virtually any other regrettable consequence of technology. Science, however, is not synonymous with technology. A science teacher with any hope (in Bruner's words) to "enlist the natural energies that sustain spontaneous learning" of science, must counter this negative image with a more accurate depiction of science as a valuable component of human culture. There are several steps to doing this.

First, there must be some sense of historical perspective illustrating that science has long been a significant and beneficial part of human culture. The observations, experiments, laws, and principles of science were obtained by the efforts of specific people to solve specific problems. What were those problems? Of what intellec- 
tual or practical significance was it that they be solved? Who attempted to solve them? What methods were tried? What difficulties were encountered? What were the seminal contributions of those to whom discoveries are attributed? How, to the extent one can know, did these people arrive at their conclusions? Addressing questions like these give students a sense of the intellectual struggle and human creativity involved in scientific discovery. Science becomes a human endeavour, a quest for answers, not merely a collection of technical facts. This is important because nonscientists (including many science teachers) unfamiliar with the nature of science envision the laws of nature as simply being "out there" ready for picking like fruit from a tree. Textbooks often foster that image by presenting experiments, data, equations, and conclusions all neatly ordered, devoid of the drama of human effort. But this is not how our understanding of the physical world came about, nor how science works today.

Newton's laws of motion and law of gravity, for example, are among the enduring topics that will forever be a part of the introductory physics curriculum. Over the years many a student left glassy eyed from calculating trajectories of falling cannon balls or orbiting satellites doubtless wondered "Why bother?" However, I have personally found that when students understand more of the circumstances of Newton's discoveries - that Newton addressed "the great unanswered question confronting natural philosophy" of his time (Westfall, 1980), or that even Newton had trouble initially with circular motion and centripetal acceleration (since he was, after all, discovering these concepts, not reading them from a textbook) - they look with renewed interest upon the subject. Students can be helped to understand that the laws of motion and of gravity are not academic exercises to improve their proficiency in calculation; rather they are a valuable part of humanity's intellectual heritage, a historical landmark in mankind's progress away from error, ignorance, and superstition.

Second, there must be some sense of utility. In an age dominated by technological innovation, a person ignorant of basic scientific principles and experimental skills is at a disadvantage not only in seeking gainful employment, but also in distinguishing what may be true and helpful from what is false and deceptive in the unremitting barrage of advertising and advocacy from every communication medium. People with no scientific background are easy prey to the influence of wildly improbable speculation, disingenuous marketing, occultism, mysticism, religious extremism, bogus science, and political indoctrination. There is survival value to science, and students who understand how science bears on their lives directly and affects their future well-being will have greater incentive to study it.

Third, there must be some sense of beauty. Beyond the mere utilitarian there is an aesthetic dimension of science, a "rhythm and a pattern between the phenomena of nature which is not apparent to the eye, but only to the eye of analysis" (Feynman, 1965). These aesthetic elements have long been a source of personal pleasure and intellectual stimulation for scientists. Consider, for example, physics Nobel Laureate Subrahmanyan Chandrasekhar's response to a consequence of Einstein's theory of general relativity (Curtin, 1982):

In my entire scientific life...the most shattering experience has been the realization that an exact solution of Einstein's equations of general relativity...provides the absolutely exact representation of untold numbers of massive black holes that populate the universe. This "shuddering before the beautiful", this incredible fact that a discovery motivated by a search after the beautiful in mathematics should find its exact replica in Nature, persuades me to say that beauty is that to which the human mind responds at its deepest and most profound.

Students, like scientists, will also respond to a mathematically elegant theory or masterfully designed experiment if they can understand it. The recognition of beauty in science, as in art and music, requires a prepared mind. This is a matter of positive feedback: the beauty of a subject increases with comprehension; the motivation to comprehend is stimulated by beauty. Science teachers must illustrate, wherever possible and at an appropriate level of complexity, those symmetries and patterns of nature that make the study of science profoundly interesting.

\subsection{Science, Uncertainty, and Critical Thinking}

With rare exception, I have found that many students and teachers regard science courses and textbooks as providing material the truth of which has been established beyond doubt. Such uncritical acceptance misses the essence of science as a self-correcting search for knowledge whose foundations are never absolutely certain. Even for the archetypical science of physics in which basic principles are the most thoroughly tested and widely ap- 
plicable of any science, there are laws yet to be discovered and phenomena to be understood better.

A science teacher who understands the nature of science will try to foster in the classroom a healthy scepticism of hypotheses and model illustrations encrusted with venerability in textbooks. For one thing, textbooks can be wrong and perpetuate one another's errors, particularly in the more qualitative sciences (Gould, 1987). This can also occur in physics even at the most elementary level. In one example I know, a model academic problem ${ }^{1}$, used by textbook writers for over a hundred years to illustrate the principles of static equilibrium, is treated incorrectly in every textbook of introductory physics I have seen (Resnick \& Halliday, 1977). Since this was a problem that had important ramifications well beyond academia, I solved the problem myself and tested the solution experimentally (Silverman, 2009).

Discussion and analysis in science classes of selected scientific controversies can aid both instructor and students to understand better how real science works and to distinguish it from pseudoscience (Silverman, 1992). Appropriate case studies are to be found in almost any field of science. One instructive example that draws from such widely disparate fields as astronomy and astrophysics, chemistry, geology, and paleontology is the ongoing debate over the massive Tertiary extinctions (think dinosaurs). In the words of one geologist (Raup, 1986):

Science is not the pure, isolated endeavor that is usually depicted...It is rarely a simple process of posing hypotheses, devising experimental tests, and waiting for Yes or No answers.

Principles do not simply fall into place upon the assiduous collection of facts. Rather, the lesson drawn by another geologist from the controversy over continental drift and plate tectonics is that:

We are...more likely to be swamped by meaningless data...Piling up facts does not tell us why things behave as they do. On the contrary, the factual burden of a science varies inversely with its degree of maturity. As a science progresses particular facts become increasingly comprehensible within general statements of greater explanatory power and scope (Hallam, 1973).

Moreover, whereas scientific facts are objective statements about nature, they can nevertheless have an emotional impact on people. In the anguished lament of one opponent to the then emerging new basis of geology, "If we are to believe [the hypothesis of continental drift], we must forget everything which has been learned over the last 70 years and start all over again” (Sant, 2014). True, but science sometimes works that way.

There is a lesson in the practice of science that is of importance outside the classroom and long after the completion of formal education. In my own classroom, I try to convey to students the message that if the principles of a rigorous and methodical discipline like physics are established only to within measured uncertainties and subject to occasional revisions, then how much less certain must be what passes for truth or dogma in other areas of human activity. Whether they become scientists or not, students can be helped to appreciate that a robust scepticism coupled with a need for proof is a useful mental attitude to have in the world at large.

I also stress, however, that the qualities of open-mindedness and objectivity in science should not be construed to mean all ideas are deserving of equal consideration, an anarchistic view of some philosophers (Feyerabend, 1975b) that puts science on par with astrology, witchcraft, magic, mysticism, and religion as equivalent paths to knowledge. Sophistry of this kind, if ever widely believed, poses a danger to civilised society. For if there are no objective facts and principles of nature, then there would be no cause or inclination to search for them. Such dangers are not empty rhetoric. In the US and increasingly abroad as well, efforts of religious fundamentalists to convince the public that scripture should replace real science as a mode of inquiry into natural phenomena have impeded the dissemination of scientific knowledge in public schools. Particularly in the cross-hairs are sciences like biology, geology, and physics that deal with origins and evolution, whether of species, the Earth, or the cosmos.

Scientists may differ strongly with one another over the interpretation of observations and the validity of theories; they may argue over whether ultimate truths in nature are attainable or even exist. But scientists, if they are indeed to be considered such, do not forgo the use of reason and empirical facts in the quest for knowledge. Science teachers must help students to realise the broader advantages of their science studies - that, beyond the factual details and experimental methods they are learning to use their minds to free themselves from credulity,

\footnotetext{
${ }^{1}$ The erroneous assumption of zero friction between a ladder and a wall is found ubiquitously in general physics textbooks and on the internet. The predicted forces disagree with results of actual measurements.
} 
dogma, and the primacy of wishful thinking over evidence in shaping their views of reality.

\subsection{Participation in Science}

All science is at root an empirical activity. The basic principles are not obtained from stone tablets, meditation, or textbooks, but by the creative interaction between theory and the facts that emerge from observation and controlled experiment. Science teachers generally realise that some participation in scientific activities is necessary for students, and include instructional laboratory work along with lectures, particularly in the introductory science courses.

An instructional laboratory can be useful in introducing students to standard apparatus and measuring techniques. However, as usually constituted, it does not represent what scientists experience when they are performing experimental research. Science instructors deceive themselves if they believe that any laboratory exercise designed from the outset to yield clean, unambiguous data in a reasonably short time on previously well-studied phenomena with low probability of failure could in any serious way reflect what experimental science is like.

Nor are students oblivious to the distinction between cookbook laboratory work and real science. Introductory laboratories are often cited as the most tedious part of a science course. It is there, perhaps more overtly than in any other part of formal coursework that assignments are purely academic exercises that demand of the performers only the repetition of well-established procedures that do not inspire, or perhaps even permit, innovation, and that lead in the end to results of no interest to anyone outside the classroom.

Yet it is the experimental aspect of science-the planning, looking, touching, manipulating, controlling, measuring, recording, checking - this direct contact with the phenomena of nature for the purpose of satisfying one's own curiosity that has provided many scientists their strongest motivation and deepest satisfaction. A discovery need not be of momentous general significance to science as long as it is personally meaningful to the discoverer. Consider, for example, the reaction of British naturalist Alfred Russell Wallace to a rather small discovery (Beverage, 1950).

None but a naturalist can understand the intense excitement I experienced when at last I captured it [new species of butterfly]. My heart began to beat violently, the blood rushed to my head, and I felt much more like fainting than I have done when in apprehension of immediate death...so great was my excitement produced by what will appear to most people a very inadequate cause.

Teachers who embrace a perspective of science consistent with how science is practised will understand that the experience and enjoyment of science require active participation, not passive absorption-participation, moreover, in a pursuit of answers to real questions. They will realise that the opportunity to pursue an authentic research activity —not necessarily for publication, but simply for the heady experience of exploration and problem solving — can provide incentive to students as it has for centuries to scientists. Teachers who are themselves experimental scientists can make such opportunities available to students by engaging them in original research projects in their laboratories. Opportunities like these, however, do not exist everywhere, nor would all students wish to take advantage of them. But an enterprising teacher at any level of science instruction can always find some way to involve students, individually or in small groups, in meaningful scientific investigations. Scheduled laboratory time, itself, can be used for this purpose to greater educational profit than for the ritual treading of ancient pathways.

Over a long career of teaching physics, I have found a number of ways to accommodate within the structure and time constraints of my courses activities that expose students to contemporary advances in science beyond the textbook and classroom lectures (Silverman, 1995). For example:

- Keeping a physics journal: Throughout the academic term students are required to keep within a bound notebook a weekly account of newsworthy items relating to advances in physics pertinent to the course. A list of suggested sources comprehensible to an educated layman was provided, which included international science publications (e.g. Nature, Science, Scientific American), websites of science organisations (e.g. American Physical Society, Institute of Physics), websites of national and international laboratories (e.g. Fermilab, CERN [European Organisation for Nuclear Research], NASA [National Aeronautics and Space Administration]), and selected major metropolitan newspapers (e.g. New York Times, Washington Post, The Guardian) noted for reporting of scientific events. A minimum of two entries per week is expected. On the last class day of the week (usually a Friday), time would be made available in class for students to share and 
discuss their journal entries. When appropriate, I would clarify a student’s report, answer questions from the class, and show how the article ties in to the formal material of the course.

- Researching and reporting on an assigned topic: A course of lectures in which the instructor does all the speaking and the students sit passively taking notes can be tedious and uninspiring. In nearly every course I teach, students are required to choose (around the middle of the term) a well-defined issue, relevant to the course, which raises questions that interest them. They research, either individually or as part of a two- or three-person panel, the topic during the remainder of the semester, and submit a formal research report at the end on the results of their investigation. Moreover, time is also made available for the students to give computer-aided oral presentations of their projects to the entire class. Although many students do not need assistance is finding topics, a list of suggestions is provided at the beginning of the term. The array of topics will vary, depending on the subject matter of the course. A sample of topics recently investigated by students in a section of my calculus-based course on introductory physics devoted to physics of the $20^{\text {th }}$ and $21^{\text {st }}$ centuries included such questions as:

- Do particles of light (photons) have mass?

- Is it really the case that neutrinos easily pass through the entire Earth?

- Why is there more matter than anti-matter in the universe?

- Are there magnetic monopoles in nature?

- What is the origin of gamma ray bursts?

- How does the global positioning system (GPS) work?

- What is a quantum computer, and can one actually be constructed?

- What causes coloured halos around the Moon or Sun?

- Why is the sky dark blue at the zenith and reddish orange at the horizon?

Note that the foregoing brief sample of topics includes exotic subjects that students do not ordinarily encounter in their daily lives (e.g. gamma rays or neutrinos) as well as relatively familiar things that a student may often use, see or hear about (GPS device or sky colour) but not really understand.

- Attending physics seminars: Besides in-class activities designed to heighten students' awareness of, and interest in, physics as a live and progressing field of study (and not a moribund discipline captured in a textbook), students in my classes are encouraged to attend public lectures on physics by guest speakers. As the organiser of a programme of public lectures, I try to invite scientists who are particularly skilled at communicating with undergraduate students. The seminars are given at the end of the week at a time that minimally conflicts with other student activities, and those who attend are accorded some measure of extra credit as an incentive. In the initial few minutes of the first class of the following week, time is devoted to discussing the content of the seminar.

Besides exposing students to contemporary scientific research, a significant feature of the three preceding activities is that they provide diverse opportunities for students to write and speak. This, in my opinion, is especially important. In the traditional science or engineering curriculum at a US university or college, students may be required to take one course ("freshman seminar") during their first term in which they have writing assignments - but afterward may never have to write or speak again in class. And yet, to be able to write clearly and speak articulately are perhaps the two most important skills a person will need for a successful career.

Another feature to be highlighted is that the foregoing activities, in place of frequent testing, which ordinarily occupies most of the non-lecture time in a US science classroom, fosters self-improvement and cooperation, rather than competition, among students. This is a significant virtue because test-taking is a skill rarely if ever needed outside academia, whereas the ability to work well in a group setting is critical to success in many science and non-science careers. Also, as I have discussed elsewhere (Silverman, 2015), by replacing the emphasis on testing (which at best measures what a student knows at a particular moment in time) with activities that involve research, writing, and speaking (which allow students to show what they have accomplished over an interval of time), an instructor can reduce the motivation for students to cheat.

\section{Concluding Remarks}

The methods of motivating science learning discussed throughout this article are all moulded from the same metal: that science instruction is more efficacious and enduring when it reflects the intrinsic activities practised by active scientists themselves. Science is not a stockpiling of phenomenological detail, but a self-correcting 
mode of inquiry pursued by people who are curious about the natural world and will satisfy their need for rational answers to personally meaningful questions.

To teach science well, one needs to have the attitudes of a scientist: to see science as a culturally important activity, technically useful and aesthetically moving; to understand that science, more than any other human activity, helps free the mind from ignorance, superstition, prejudice, and dogma; to have the curiosity oneself, to explore natural phenomena - at whatever level one is capable of, and by whatever means one has available-and to enjoy sharing this curiosity with students.

The science teacher whose instruction goes no further than the textbook, whose notes have yellowed with age from unvarying use, and whose concept of scientific experience is ritualised repetition of procedure cannot hope to motivate and inspire students. Teachers must, themselves, be motivated and inspired: to read avidly and regularly in order to learn lessons of the past and keep abreast of the present; to experiment and tinker in order to teach with confidence based on personal experience; to try in all ways possible to make the science classroom reflect accurately the attributes and activities of a place where real science is done.

\section{References}

Berland, T. (1962). The Scientific Life (pp. 65-66). New York: Coward-McCann.

Beverage, W. I. B. (1950). The Art of Scientific Investigation (pp. 192). New York: Vintage Books.

Bruner, J. S. (1966). Toward a Theory of Instruction (pp. 115). Cambridge: Harvard University Press.

Bulwer-Lytton, E. G. (1873). Kenelm Chillingly: His Adventures and Opinions (Chap. 12). http://www.gutenberg.org/files/7658/7658-h/7658-h.htm\#link2HCH0001

Conover, E. (2015). Why Did You Become a Scientist? Researchers Speak Out. Science http://news.sciencemag.org/scientific-community/2015/04/why-did-you-become-scientist-researchers-speak-out-iamascien $\underline{\text { tistbecause }}$

Curtin, D. (1982). The Aesthetic Dimension of Science (pp. 7). New York: Philosophical Library.

Feyerabend, P. (1975a). How to Defend Society against Science. Radical Philosophy, 11, 3-8.

Feyerabend, P. (1975b). Against Method: Outline of an Anarchistic Theory of Knowledge. New York: New Left Books.

Feynman, R. P. (1965). The Character of Physical Law (pp. 13). Cambridge: MIT Press.

Fraiberg, S. (1959). The Magic Years. New York: Charles Scribner.

Gould, S. (1987). The Case of the Creeping Fox Terrier Clone. Natural History, 97, 16-24.

Hallam, A. (1973). A Revolution in the Earth Sciences (pp. 112-113). Oxford: Oxford University Press.

Mahon, B. (2004). The Man Who Changed Everything: The Life of James Clerk Maxwell. New York: Wiley.

Neuman, S. (2014). 1 in 4 Americans Thinks the Sun Goes around the Earth, Survey Says. National Public Radio. http://www.npr.org/sections/thetwo-way/2014/02/14/277058739/1-in-4-americans-think-the-sun-goes-around-the-earth-su rvey-says

O’Neill, I. (2014). 1 in 4 Americans Don’t Know Earth Orbits the Sun. Yes, Really. http://news.discovery.com/space/astronomy/1-in-4-americans-dont-know-earth-orbits-the-sun-yes-really-140214.htm

Raloff, J. (2010). Science Literacy: U.S. College Courses Really Count. Science News. https://www.sciencenews.org/blog/science-public/science-literacy-us-college-courses-really-count

Raup, D. (1986). The Nemesis Affair (pp. 18). New York: Norton.

Resnick, R., \& Halliday, D. (1977). Physics (3rd ed., pp. 288-289). New York: Wiley.

Sant, J. (2014). Wegner and Continental Drift Theory. http://www.scientus.org/Wegener-Continental-Drift.html

Silverman, M. P. (1992). Raising Questions: Philosophical Significance of Controversy in Science. Science \& Education, 1, 163-179. http://dx.doi.org/10.1007/BF00572837

Silverman, M. P. (1995). Self-Directed Learning: A Heretical Experiment in Teaching Physics. American Journal of Physics, 63, 495-508. http://dx.doi.org/10.1119/1.18080

Silverman, M. P. (2009). Final Report: Force of a Ladder on a Railing under Static and Dynamic Conditions. Expert Witness Testimony (Used by Both Defendant and Plaintiff) in the Case Shattuck v. Wynfield, Hartford Superior Court (Hartford CT).

Silverman, M. P. (2015). Cheating or Coincidence? Statistical Method Employing the Principle of Maximum Entropy for Judging Whether a Student Has Committed Plagiarism. Open Journal of Statistics, 5, 143-157.

http://dx.doi.org/10.4236/ojs.2015.52018 
Sreenivasan, K. R. (2004). One Hundred Reasons to Be a Scientist (pp. 1-268). Trieste: ICTP (Abdus Salam International Centre for Theoretical Physics).

Watts, A. (1970). Does It Matter (pp. 25)? New York: Vintage Books.

Westfall, R. (1980). Never at Rest: A Biography of Isaac Newton (Chap. 10, pp. 402-406). Cambridge: Cambridge University Press. 